\title{
Industrie du jeu vidéo et construction de clusters innovants
}

\section{Bruno Lusso}

\section{(2) OpenEdition}

1 Journals

Édition électronique

URL : https://journals.openedition.org/sdj/848

DOI : $10.4000 /$ sdj. 848

ISSN : 2269-2657

\section{Éditeur}

Laboratoire EXPERICE - Centre de Recherche Interuniversitaire Expérience Ressources Culturelles Education

\section{Référence électronique}

Bruno Lusso, «Industrie du jeu vidéo et construction de clusters innovants », Sciences du jeu [En ligne], 8| 2017, mis en ligne le 26 décembre 2017, consulté le 15 septembre 2021. URL : http:// journals.openedition.org/sdj/848; DOI : https://doi.org/10.4000/sdj.848

Ce document a été généré automatiquement le 15 septembre 2021.

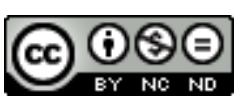

La revue Sciences du jeu est mise à disposition selon les termes de la Licence Creative Commons Attribution - Pas d'Utilisation Commerciale - Pas de Modification 4.0 International. 


\title{
Industrie du jeu vidéo et construction de clusters innovants
}

\author{
Bruno Lusso
}

1 «Le marché du jeu vidéo se porte extrêmement bien. C'est la quatrième année consécutive de croissance et on dépasse le précédent pic de 2008 qui a marqué l'apogée de la génération 7 des consoles " s'est réjouie Julie Chalmette, présidente du Syndicat des éditeurs de logiciels de loisirs au cours d'une conférence de presse présentant le bilan de l'industrie du jeu vidéo française pour l'année 2016. Le marché du jeu vidéo français a atteint en 2016 un chiffre d'affaires record de 3,46 milliards d'euros, soit une croissance de $4 \%$ par rapport à l'année 2015 (SNJV-IDATE, 2016). L'industrie du jeu vidéo désigne un secteur d'activités économiques allant de la conception, à la production et à la commercialisation (sur un support ou de manière dématérialisée) d'un contenu vidéoludique. Elle regroupe à la fois la création, la production et l'intégration de contenus et de technologies, le développement de logiciels et d'applications, et l'édition et la diffusion de ces contenus numériques. S'appuyant sur des techniques aussi diverses que l'image, le son ou le traitement de données, le jeu vidéo est une industrie à fort contenu technologique qui nécessite d'étroites relations avec les structures d'enseignement supérieur et de recherche, ainsi que divers prestataires de services. Du point de vue des compétences professionnelles, cette industrie converge avec des secteurs d'activités créatives plus ou moins proches comme le cinéma, la télévision, l'animation, le web design ou les télécommunications (Lusso, 2011).

2 La proximité et la coopération entre les acteurs du jeu vidéo deviennent un enjeu majeur pour le développement de cette industrie qui s'appuie à la fois sur l'exploitation de la créativité humaine et l'innovation technologique. La mise en place de clusters apparaît comme un objectif de développement à atteindre pour les acteurs du jeu vidéo (Lusso, 2011). D'après le site internet du réseau France Clusters, un cluster désigne un réseau principalement constitué de petites et moyennes entreprises. Territorialement ancrées et œuvrant dans un même domaine d'activités, ces firmes fédèrent leurs compétences pour réaliser des produits plus innovants et conquérir de nouveaux 
marchés. Cette définition courte et quelque peu simpliste a néanmoins pour avantage de présenter les principaux enjeux économiques et territoriaux de la notion de cluster.

Figure 1 : Répartition des firmes du jeu vidéo en France

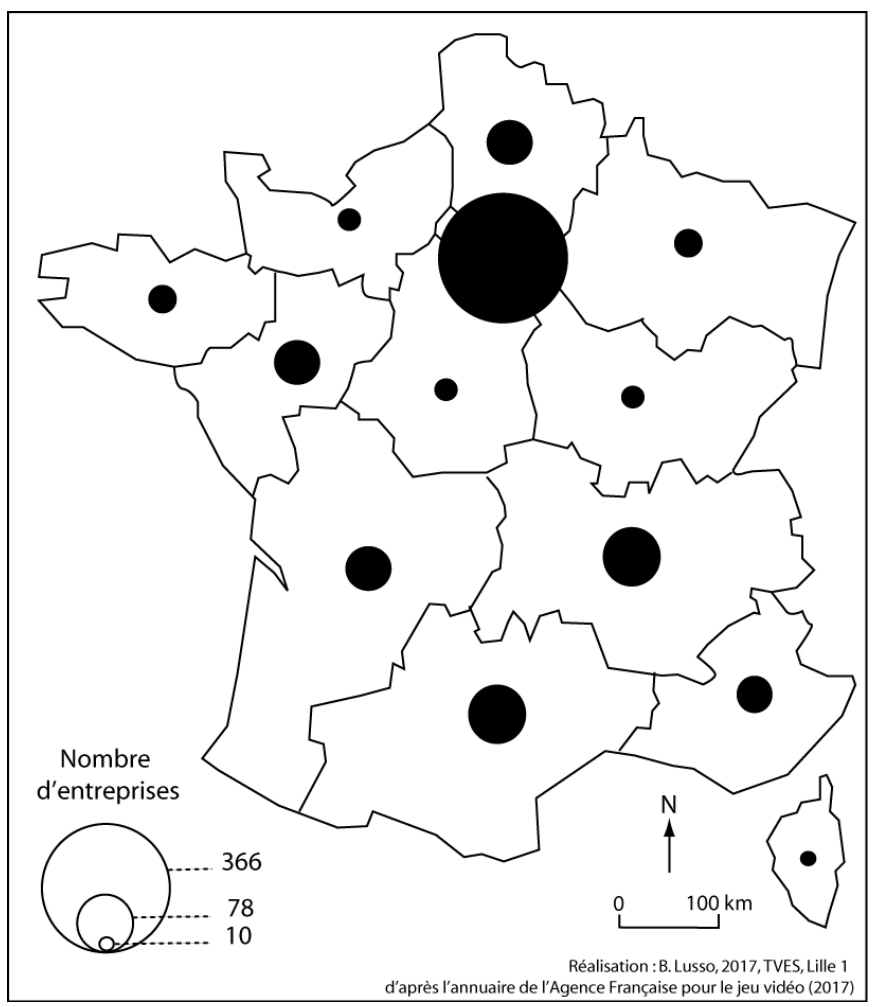

3 A l'échelle du territoire français, la production de jeux vidéo est principalement concentrée en région parisienne (Figure 1). L'Ile de France concentre deux tiers des entreprises productrices de contenus vidéoludiques. Plusieurs pôles secondaires émergent sur le territoire français comme l'Occitanie, l'Auvergne-Rhône-Alpes, les Hauts-de-France et PACA. Le territoire français est désormais composé d'un maillage fort d'entreprises du jeu vidéo réunies au sein de clusters régionaux comme Capital Games pour Paris et l'Ile-de-France, Atlangames pour la Bretagne et les Pays de la Loire. Certaines associations œuvrent même à l'échelle métropolitaine comme Bordeaux Games (SNJV-IDATE, 2016).

4 A l'échelle métropolitaine, les villes de Lyon, Lille et Marseille occupent une place essentielle en devenant, certes loin derrière Paris, des foyers de production vidéoludique à part entière. Ces trois aires métropolitaines ont mis en place une stratégie de développement du jeu vidéo originale. Les acteurs du jeu vidéo sont intégrés à des associations professionnelles plus larges comprenant des industries culturelles et créatives convergentes comme le cinéma, l'audiovisuel, l'animation, les télécommunications ou le web design.

Nous partons de l'hypothèse que les pouvoirs publics régionaux et métropolitains joueraient un rôle déterminant dans le rapprochement des acteurs de l'industrie du jeu vidéo. Ils encourageraient et financeraient la construction d'associations professionnelles thématiques et/ou spécialisées qui mettraient en œuvre une stratégie de développement qui reposerait sur la construction d'un cluster fondé sur l'innovation. Comment s'est construite l'intégration des acteurs du jeu vidéo au sein de 
structures professionnelles? Quelles sont les retombées de ces dynamiques sur le développement de l'industrie du jeu vidéo ? Après avoir présenté le cadre théorique, territorial et méthodologique de la recherche, nous étudierons les étapes conduisant au rapprochement entre les acteurs du jeu vidéo dans les trois aires métropolitaines et à la constitution d'associations professionnelles et nous terminerons par l'analyse des actions menées par ces associations professionnelles et la nature des collaborations qui se mettent en place au sein de l'industrie du jeu vidéo.

\section{L'industrie du jeu vidéo, une activité reposant sur différentes formes de proximités}

6 Dans un article paru en 2005, les géographes A-J. Scott et F. Leriche rappellent que les grandes métropoles offrent les conditions optimales au développement des industries culturelles et créatives. La concentration géographique et la possibilité de constituer des réseaux facilitent la circulation des informations et des innovations, et l'essor de ces industries (Scott et Leriche, 2005, p. 210). Ces différentes constatations nous amènent à poser la question du rôle des proximités et d'une dynamique de cluster dans le développement de l'industrie du jeu vidéo.

\section{Clusters innovants}

7 En 1998, dans son article Clusters and the new economics of competition, M. Porter associe la notion de cluster à une organisation industrielle spatialisée reposant sur des liens entre producteurs et fournisseurs en termes de proximité géographique, de complémentarités et de confiance. Les firmes colocalisées développent des relations à la fois concurrentielles et coopératives. Cette combinaison d'éléments induit un meilleur apprentissage, une diffusion de l'innovation plus forte et une plus grande compétitivité des firmes localisées dans un cluster. Cette approche a priori simple et convaincante a été en partie reprise dans les travaux de chercheurs comme M. Storper (1997) ou P. Cooke (2001). Pourtant, d'importantes zones d'ombres persistent autour des délimitations, des conditions d'émergence et d'évolution du cluster, ainsi que des domaines d'activités pouvant être touchés par une dynamique réticulaire (Leducq et Lusso, 2011). 
Figure 2 : Les attributs du cluster innovant

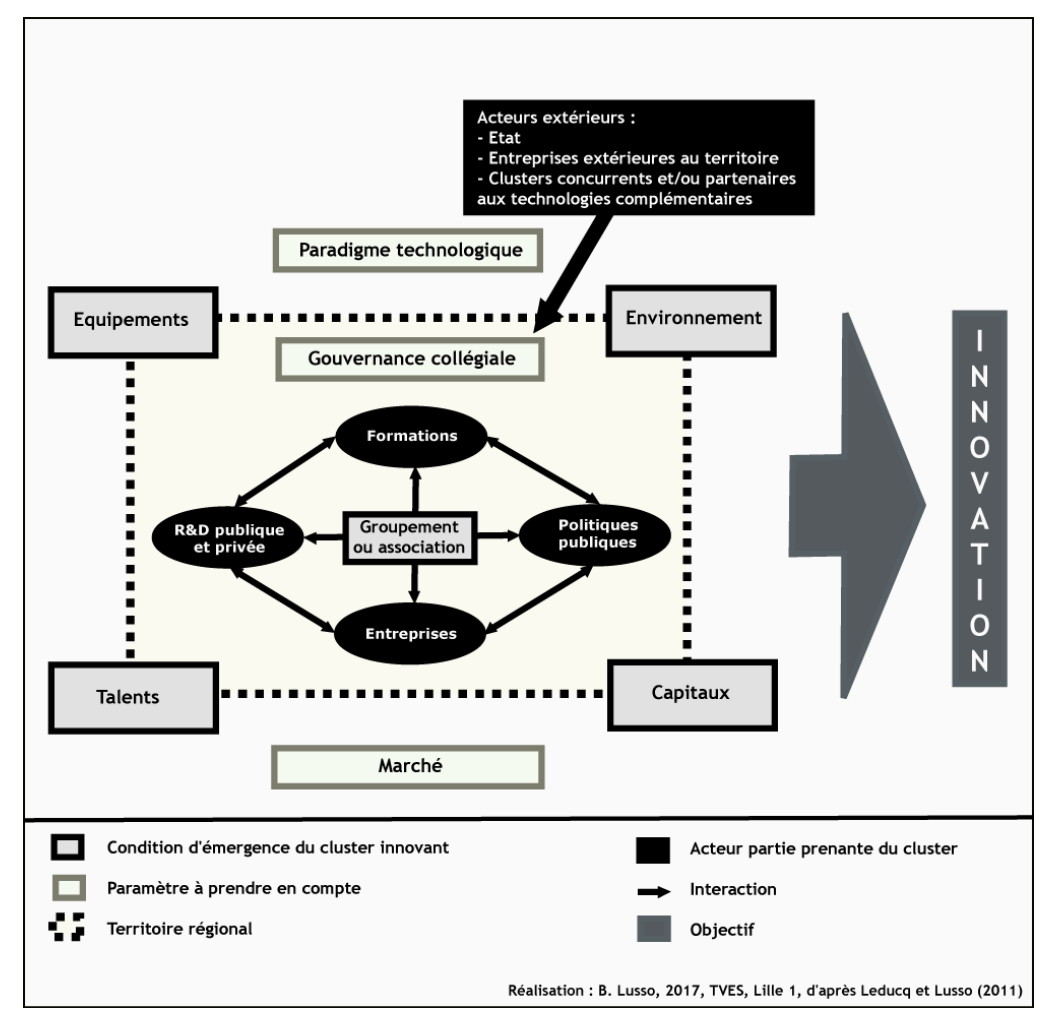

8 La question de la spécificité des clusters et des réseaux d'innovation a fait l'objet de recherches empiriques dans le secteur des hautes technologies (Saxenian, 1994; Feldman, 2003 ; Depret et Hamdouch 2009). Ces travaux ont développé une approche plus précise et opérationnelle de la notion de cluster. Le terme de cluster innovant a émergé de ces travaux (Ernst, 2006 ; Depret et Hamdouch, 2009 ; Leducq et Lusso, 2011).

Si la qualité de l'environnement local ou la présence de talents restent des conditions d'émergence du cluster innovant, celui-ci s'apparente davantage à la collaboration étroite entre les différents acteurs $d u$ développement territorial: entreprises, structures de formation, instituts de recherche publique et privée, et pouvoirs publics (Etzkowitz et Leydersdorff, 1997 ; Etzkowitz et Ranga, 2011). Ces quatre catégories d'acteurs regroupées sous la forme d'associations ou de manière plus informelle doivent se mettre en accord autour d'une stratégie ou d'une trajectoire de développement cohérente et définie de manière collégiale (Berthinier-Poncet, 2014) à l'échelle régionale (Resbeut et Gugler, 2016), afin de mieux renforcer la résilience du cluster face aux chocs extérieurs. Pouvant être animé par une entreprise leader (Gilly, Kechidi et Talbot, 2014) ou soutenu par une institution publique (Champenois, 2012), le cluster doit néanmoins rester ouvert sur le monde extérieur, afin qu'il puisse s'approprier des technologies convergentes provenant de clusters concurrents et continuer à proposer des produits répondant aux attentes du marché (Delgado, Porter et Stern, 2014). Ceci suppose des apports financiers conséquents. Le cluster innovant n'a pas une vocation immédiatement marchande (Potter et Doug-Watts, 2011) et laisse supposer des dynamiques évolutives (Crevoisier, 2001). 
10 Ainsi, selon Ron Boschma (2004), cinq grandes catégories de proximité se dégagent et deviennent nécessaires à la pérennisation d'un cluster fondé sur l'innovation, à savoir :

- La proximité institutionnelle qui peut être définie comme un espace regroupant différents acteurs autour de règles communes (Coris, 2008) et permettant de réguler les conflits (Zentelin, 2015).

- La proximité organisationnelle qui renvoie à la coordination des échanges de connaissances entre les acteurs d'un espace doté d'une structure opérationnelle favorisant l'émergence d'innovations diverses (Dubouloz et Bocquet, 2013).

- La proximité géographique qui désigne une agglomération permanente en un même endroit d'acteurs et de ressources nécessaires au fonctionnement du cluster. Mais cette proximité ne se réduit pas à un aspect purement spatial (Martinus et Sigler, 2017). Le recours aux technologies de l'information et de la communication peut alors réduire la distance entre des acteurs géographiquement éloignés (Brennan et Martin, 2012).

- La proximité sociale, une notion qui provient de la littérature liée à l'encastrement (Granovetter, 1985) et qui souligne l'importance du contexte social dans la constitution de réseaux. Cette notion est à distinguer de la proximité culturelle qui renvoie au partage de valeurs communes en relation avec une communauté et facilitant la construction de réseaux (Saxenian, 2006).

- La proximité cognitive qui est liée à un groupe de personnes partageant la même base de connaissances et de compétences, et pouvant apprendre les unes des autres (Boschma, 2004 ; Huber, 2011).

11 Ces cinq grandes catégories de proximités jouent un rôle déterminant dans la réussite du cluster innovant qui peut s'appliquer à l'industrie du jeu vidéo.

\section{Panorama de l'industrie vidéoludique dans les trois aires métropolitaines}

D'après l'Annuaire de l'Association française pour le jeu vidéo (2017), les aires métropolitaines de Lille, de Lyon et de Marseille comptent respectivement 29, 50 et 15 entreprises productrices de contenus vidéoludiques. 
Figure 3 : Distribution des entreprises du jeu vidéo selon leur domaine d'activité principal dans les aires métropolitaines de Lille, Lyon et Marseille

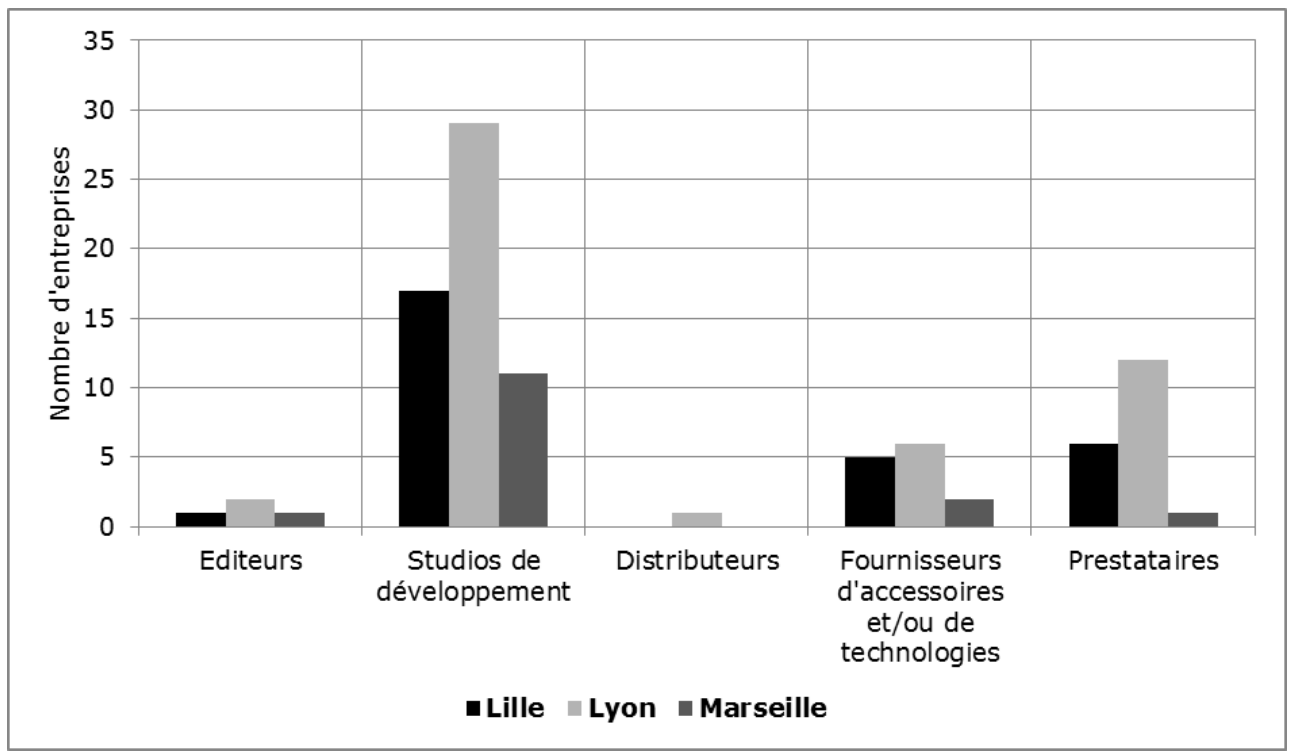

Réalisation : B. Lusso, 2017, TVES, Lille 1, d'après l'Annuaire de l'Association française pour le jeu vidéo (2017)

13 Avec plus de la moitié du nombre d'entreprises, les studios de développement constituent le principal acteur de l'industrie du jeu vidéo. Le développeur possède son propre studio, recrute et coordonne des professionnels techniques et artistiques chargés de concevoir et de réaliser un jeu vidéo. Pour ce faire, il peut s'appuyer sur des fournisseurs d'accessoires, de technologies ou de logiciels qui représentent entre un cinquième (Marseille) et un tiers (Lille et Lyon) du nombre total de firmes productrices de contenus vidéoludiques. Quant aux distributeurs et aux éditeurs (qui assurent la publication et la diffusion du jeu vidéo), ils sont beaucoup plus rares, soit une à trois entreprises dans chacune des aires métropolitaines. 
Figure 4 : Répartition des entreprises productrices de jeux vidéo dans les aires métropolitaines de Lille, Lyon et Marseille

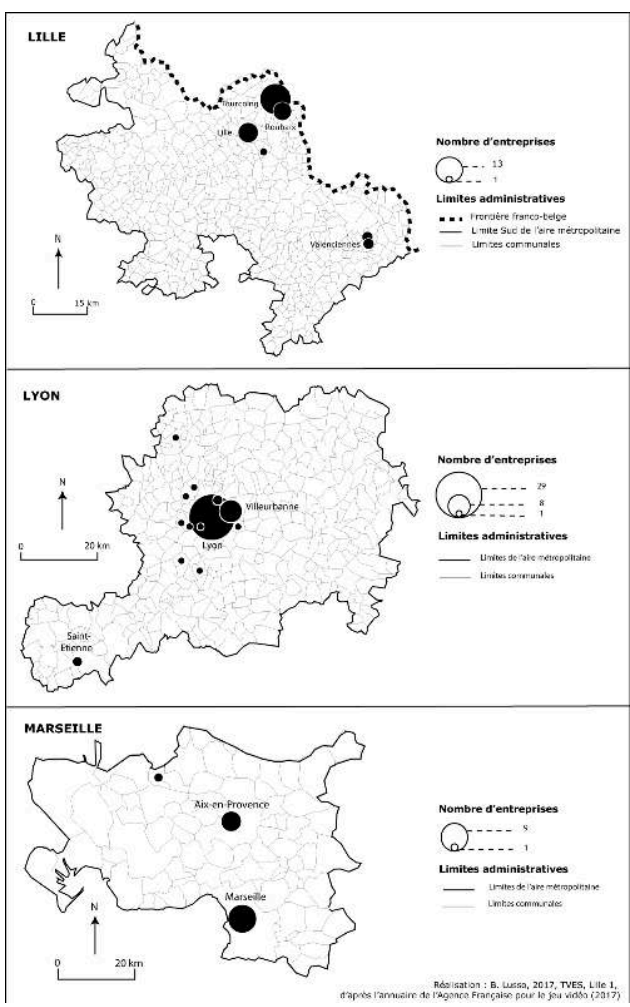

14 D’un point de vue spatial, les entreprises du jeu vidéo privilégient les grands centres urbains des aires métropolitaines (villes de Lille, Lyon, Marseille et Aix-en-Provence). Malgré tout, quelques pôles existent en banlieue (commune de Villeurbanne pour Lyon, villes de Roubaix et Tourcoing pour la métropole lilloise) ou dans des agglomérations secondaires des aires métropolitaines (Valenciennes pour Lille, Saint-Etienne pour Lyon). Cette première lecture montre que la ville demeure un espace privilégié pour le développement de l'industrie du jeu vidéo. 
Figure 5 : Distribution des entreprises productrices de contenus vidéoludiques selon le nombre de salariés dans les aires métropolitaines de Lille, Lyon et Marseille

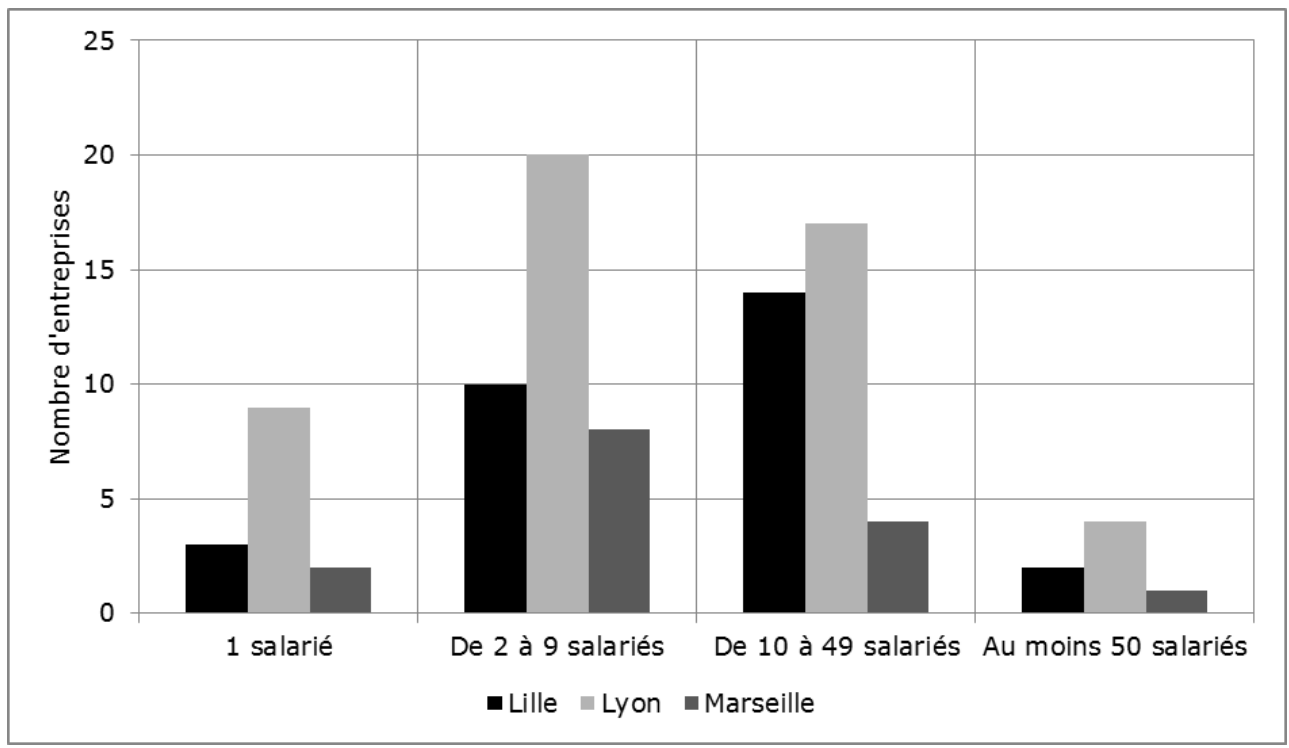

Réalisation : B. Lusso, 2017, TVES, Lille 1, d'après l'Annuaire de l'Association française pour le jeu vidéo (2017)

15 Les TPE/PME productrices de contenus vidéoludiques restent dominantes dans les trois aires métropolitaines. Si les entreprises de moins de 10 salariés représentent un peu moins de $50 \%$ du tissu entrepreneurial à Lille, les proportions dans les aires métropolitaines de Lyon et de Marseille sont plus importantes avec respectivement $58 \%$ et $67 \%$ d'entreprises de moins de 10 salariés. A l'exception de l'exemple lillois, les PME restent minoritaires. Quant aux firmes de plus de 50 salariés, elles ne dépassent dans aucune des aires métropolitaines étudiées la proportion de $8 \%$.

Déficitaires en ressources financières et en capacité de coopération (Alberti, 2004), les TPE/PME - nombreuses au sein de l'industrie du jeu vidéo des aires métropolitaines de Lille, Lyon et Marseille - adoptent des comportements individualistes (Mendez, Bardet et Courault, 2008) qui nuisent à la constitution de réseaux collaboratifs. Pourtant, le rapprochement des acteurs du jeu vidéo est indispensable. Pour les pouvoirs publics, la notion de cluster innovant est devenue depuis les années 1990 un outil de dynamisme économique et de développement des territoires. A l'échelle mondiale, l'OCDE (1999) définit les clusters innovants comme des réseaux de production dans lesquels les firmes, les universités, les instituts de recherche, les investisseurs et les institutions bancaires jouent un rôle structurant. Le principal intérêt de l'approche de l'OCDE consiste dans l'apport indispensable de ressources financières au cluster, qu'elles soient d'origine publique ou privée. En Europe, ces initiatives sont relayées par la Commission européenne qui a lancé un projet d'Observatoire européen des clusters. Son objectif est de suivre la dynamique des clusters les plus innovants afin d'établir une méthodologie commune à l'Union européenne (Leducq et Lusso, 2011).

En France, la politique de soutien aux clusters les plus innovants a débuté avec la labellisation par l'État en 2005 de 71 pôles de compétitivité chargés de soutenir le développement des réseaux industriels entre les firmes et les centres de recherche. Disposant de compétences économiques plus larges depuis les politiques de décentralisation des années 1980, les Conseils Régionaux (puis les régions) et les 
communautés urbaines (puis les métropoles) se sont inspirés des principes de la politique nationale pour labelliser, avec une enveloppe financière moindre, leurs pôles d'excellence régionaux ou métropolitains. C'est dans ce cadre que les pouvoirs publics des aires métropolitaines de Lille, Lyon et Marseille ont encouragé la création d'associations professionnelles rapprochant les acteurs du multimédia (audiovisuel, animation, jeu vidéo, internet et télécommunications) : Imaginove à Lyon dès 2007, Pôle Images Nord-Pas de Calais (Pictanovo à partir de 2013) à Lille en 2009 et Pôle Régional de l'Image, du Multimédia et de l'Internet (PRIMI) à Marseille en 2010. Ces différentes associations professionnelles ont été labellisées pôle d'excellence régional par le Conseil Régional du territoire dans lequel elles se localisent et pour Imaginove, par l'État dans le cadre de sa politique menée en faveur des pôles de compétitivité.

18 Ces associations professionnelles se sont progressivement construites et ont mis en place des actions visant à animer l'industrie du jeu vidéo régionale. Afin de connaître les modalités de mise en œuvre de ces politiques réticulaires au sein de l'industrie du jeu vidéo des aires métropolitaines de Lille, de Lyon et de Marseille, un protocole de recherche a été mis en place. Celui-ci reprend et actualise mes travaux de recherche doctorale menés entre 2007 et 2011 et portant sur les dynamiques de l'industrie du multimédia dans les aires métropolitaines de Lille, de Lyon et de Marseille (Lusso, 2011). L'identification des acteurs a constitué un préalable nécessaire à la réalisation d'entretiens semi-directifs permettant d'évaluer le degré de structuration de l'industrie du jeu vidéo dans les trois territoires. Les entretiens réalisés auprès des directions à l'économie des métropoles et des régions ont servi de base pour définir la stratégie menée en direction de l'industrie du jeu vidéo. Les échanges effectués auprès des associations professionnelles ont été utiles pour retracer les étapes de la structuration de l'industrie et identifier les actions mises en place pour rapprocher les différents acteurs du secteur. Cette première partie du travail de recherche, réactualisée et approfondie en 2017 par l'analyse des rapports annuels d'activité des associations professionnelles, a permis de déterminer le degré de proximité institutionnelle et organisationnelle au sein de l'industrie du jeu vidéo des trois aires métropolitaines. Les proximités géographique, sociale et cognitive ont pu être identifiées à partir d'entretiens effectués auprès d'éditeurs et de développeurs de jeux vidéo. Au total, 11 entreprises ont fait l'objet d'un entretien dans l'aire métropolitaine de Lille, contre 8 à Lyon et 5 à Marseille. Ces entretiens réalisés entre 2008 et 2011 ont été réactualisés et complétés par la lecture d'articles de journaux sur internet et la consultation des sites officiels de ces firmes du jeu vidéo. Ces recherches ont favorisé l'identification des différents réseaux mis en œuvre par ces entreprises, la définition des échelles géographiques et des différentes formes de collaboration (proximité géographique). Ces entretiens ont été aussi l'occasion de reconstituer le parcours des entrepreneurs et de préciser le type de relations existant au sein de l'industrie du multimédia et du jeu vidéo (proximité sociale). Enfin, la proximité cognitive a pu être évaluée lors de ces rencontres, puis par des recherches effectuées à partir des bilans d'activité des associations professionnelles. En effet, les programmes de recherche auxquels les entreprises du jeu vidéo participent ont permis d'identifier les modalités de transmission des connaissances du monde de la recherche vers les entreprises. 


\section{Le difficile rapprochement des acteurs du jeu vidéo}

19 L'objectif de cette section est de retracer les facteurs et les étapes qui ont abouti à la création d'organisations professionnelles chargées d'animer l'industrie du jeu vidéo et d'analyser la place des institutions publiques métropolitaines et régionales dans ces dynamiques. Ceci permet de définir le niveau de proximité institutionnelle et organisationnelle au sein de l'industrie du jeu vidéo des trois aires métropolitaines.

\section{Des réseaux interentreprises quasiment inexistants}

Dans les aires métropolitaines de Lille, de Lyon et de Marseille, les collaborations entre les firmes du logiciel et du jeu vidéo sont initialement très limitées. En 2011, plus des deux tiers des entreprises productrices de jeux vidéo déclaraient ne pas connaître les firmes vidéoludiques concurrentes (Lusso, 2011). Les très rares réseaux mis en œuvre se résument à une organisation verticale de la production où un éditeur demande à des entreprises sous-traitantes de produire et d'assembler le jeu vidéo. Cette organisation de la production peut déstabiliser le tissu productif lorsque l'éditeur en question est confronté à des difficultés économiques. A Lyon, le déménagement en 2012 de la société Atari à Paris, puis sa mise en faillite en 2013, ont eu des répercussions négatives sur plusieurs filiales et sous-traitants locaux. Si le studio Eden Games est redevenu indépendant en 2014 après sa fermeture par Atari en 2013, l'annulation des contrats d'Atari a été fatale à Gamr7 qui a mis la clé sous la porte en 2012. Une organisation verticale de la production ne protège pas une entreprise face aux risques liés aux variations du marché.

21 La faiblesse des collaborations dans le domaine du jeu vidéo tient aussi au rapport particulier que les entreprises entretiennent avec la R\&D (recherche et développement). Lorsqu'un éditeur de jeux vidéo met en œuvre de la R\&D, elle se développe généralement en interne, est associée à une production et prend fin lorsque cette dernière est terminée. Les courants d'innovation sont plutôt exogènes et imposés en amont par les acteurs du matériel informatique (hardware). Dans ce contexte, les producteurs de contenus vidéoludiques produisent un effort d'organisation interne conséquent en matière de R\&D par un processus de capitalisation des compétences, et notamment celle des ingénieurs et des créateurs. C'est le cas de l'entreprise lilloise de communication visuelle Ankama créée en 2001 et qui a progressivement élargi ses compétences au jeu vidéo ou à l'édition de bandes dessinées par un système de croissance en interne. La division de l'activité économique entre plusieurs départements autonomes (Ankama Games, Ankama Studio, Ankama Animation, Ankama Editions et Ankama Products) permet à l'entreprise de mieux résister aux crises auxquelles l'industrie est régulièrement confrontée, mais aussi d'être moins dépendante d'acteurs exogènes (Lusso, 2011). Une telle situation ne facilite donc pas la mise en œuvre de réseaux.

\section{Des premiers clubs d'entreprises thématiques aux associations transmédia}

La difficulté à établir des réseaux interentreprises solides et durables est une réalité de l'industrie du jeu vidéo dans les trois aires métropolitaines. Pourtant, plusieurs 
entrepreneurs ont tenté de créer dès la fin des années 1990 des clubs d'entreprises autour de l'industrie du logiciel et du jeu vidéo. C'est le cas de l'association Lyon Game qui a œuvré dès 1999 au sein de l'association des producteurs de contenus numériques Lyon Infocité pour un rapprochement entre les acteurs lyonnais du jeu vidéo. Dès 2004, le nom de Lyon Game est associé à celui de l'association Lyon Infocité. De nombreuses actions visant à animer l'industrie du jeu vidéo sont mises en place comme le lancement en 2004 du salon professionnel Game Connection, la création la même année de l'école du jeu vidéo Gamagora et du Serious Game Summit. Ces actions s'avèrent être décisives dans la structuration de l'industrie lyonnaise du jeu vidéo (Lusso, 2011).

L'expérience du club lyonnais a été suivie par d'autres projets bien plus modestes dans les aires métropolitaines de Lille et de Marseille. Dans l'agglomération lilloise, l'Arc Numérique, association créée en 2001 et constituée d'entreprises de communication visuelle, de développeurs de logiciels et de jeux vidéo, est restée une coquille vide et disparaît en 2003. Quant à l'aire métropolitaine de Marseille, la création en 2007 de l'association professionnelle Gamesud résulte de la volonté d'un studio de développement parisien, Lexis Numérique, et de trois entreprises régionales, de fédérer les professionnels du jeu vidéo. La mise en place de ce club d'entreprises apparaît comme une réponse au refus de l'association des producteurs de cinéma Pole Sud Image d'intégrer les acteurs du jeu vidéo, en dépit des injonctions de la région PACA qui souhaitait initialement rapprocher ces activités créatives. A la fin des années 2000, les aires métropolitaines de Lille et Marseille ne disposent pas d'associations professionnelles du jeu vidéo ayant engagé un ambitieux programme d'animation de la filière comme à Lyon.

Pourtant, la fin des années 2000 marque un tournant dans la structuration de l'industrie vidéoludique des aires métropolitaines de Lille, Lyon et Marseille. Cette évolution est à mettre en relation avec les mutations des technologies du multimédia marquées par une dématérialisation et une diversification des supports de diffusion (Chantepie et Le Diberder, 2012). De nouveaux formats apparaissent comme la télévision connectée, les tablettes ou les smartphones, ce qui entraîne de nouveaux usages et modes de consommation (Augros, 2012). Il existe désormais de multiples déclinaisons pour un film, allant du DVD au Blu Ray, en passant par la VOD ou la télévision HD. Dans un contexte d'essor des industries créatives et de l'économie des loisirs, la conversion d'une partie de la production cinématographique aux techniques numériques a permis de créer de larges zones de convergence entre les activités audiovisuelles et celles du jeu vidéo. L'adjectif "transmédia » émerge au sein de l'industrie du jeu vidéo et désigne une pratique consistant à développer un contenu narratif sur plusieurs médias (cinéma, audiovisuel, jeu vidéo, Internet, etc). Le contenu développé et les capacités d'interaction sont différenciés selon les spécificités de chaque média (Azemard, 2013). Ces mutations encouragent donc les firmes à privilégier désormais dans leur politique de recrutement les profils pluridisciplinaires.

C'est dans ce contexte que les entreprises du jeu vidéo des aires métropolitaines de Lille, Lyon et Marseille intègrent entre 2005 et 2010 des associations professionnelles transmédia. Le rapprochement n'a pas été automatique et a nécessité l'intervention des pouvoirs publics régionaux et métropolitains. Lors de sa création à Lyon en 2005, le pôle de compétitivité Imaginove comprenait uniquement les acteurs du jeu vidéo réunis au sein de l'association Lyon Game qui avait obtenu dès 2002 du Conseil Régional de Rhône-Alpes le label Cluster Loisirs Numériques. Au cours de l'année 2006, sous 
l'impulsion de la région, le pôle de compétitivité intègre les acteurs régionaux du cinéma, de l'audiovisuel, de l'animation et des télécommunications. Au début de l'année 2007, le pôle de compétitivité Imaginove devient une association régionale résolument transmédia. En région PACA, la situation est plus délicate. L'association Pole Sud Image, qui regroupe les producteurs de l'audiovisuel, refuse d'intégrer à sa structure les acteurs du jeu vidéo. Principal financeur de l'association, la région PACA impose le rapprochement entre les professionnels de l'audiovisuel et du jeu vidéo. Le lancement du PRIMI en juin 2010 concrétise l'intégration des acteurs du jeu vidéo à une association professionnelle transmédia. Enfin, dans l'aire métropolitaine de Lille, l'absence de structure professionnelle a facilité la tâche du Conseil Régional du NordPas de Calai qui décide de lancer en juillet 2009 une association publique régionale transmédia, le Pôle Images Nord-Pas de Calais rebaptisé Pictanovo en 2013.

\section{Les acteurs du jeu vidéo au sein des associations professionnelles transmédia}

L'intégration des acteurs du jeu vidéo au sein d'associations professionnelles transmédia a entraîné de profondes restructurations. Certains clubs d'entreprises du jeu vidéo disparaissent ou sont absorbés par les associations professionnelles transmédia. Dans l'aire métropolitaine de Lyon, l'apparition d'Imaginove a entraîné en 2008 l'intégration de Lyon Game au pôle de compétitivité. A Marseille, si Gamesud a maintenu ses activités pendant un certain temps après la création du PRIMI en 2010, elle a été progressivement absorbée par l'association professionnelle transmédia et a cessé toute activité depuis 2014. En revanche, à Lille, l'absence de structures professionnelles au sein de l'industrie du jeu vidéo a encouragé le Conseil Régional du Nord-Pas de Calais à créer parallèlement au pôle image un club d'entreprises vidéoludiques, Game In. Cette stratégie traduit la volonté de la région de construire des collèges constitués de professionnels issus des différentes filières de l'industrie du multimédia qui participeraient à l'orientation stratégique du pôle transmédia (Lusso, 2011).

Tableau 1 : Distribution des acteurs du jeu vidéo adhérant à Pictanovo, à Imaginove et au PRIMI en 2017

\begin{tabular}{|l|l|l|l|}
\hline Adhérents au pôle & Pictanovo & Imaginove & PRIMI \\
\hline Total & 157 & 128 & 128 \\
\hline $\begin{array}{l}\text { Entreprises du jeu } \\
\text { vidéo }\end{array}$ & 17 & 24 & 11 \\
\hline Ecoles & 29 & 11 & 16 \\
\hline $\begin{array}{l}\text { Laboratoires de } \\
\text { recherche }\end{array}$ & 16 & 4 & 2 \\
\hline $\begin{array}{l}\text { Clubs d'entreprises } \\
\text { du jeu vidéo }\end{array}$ & Game In & Only Game & $\begin{array}{l}\text { Gamesud (absorbée } \\
\text { par le PRIMI depuis } \\
\text { 2011) }\end{array}$ \\
\hline
\end{tabular}




\begin{tabular}{|c|c|c|c|}
\hline $\begin{array}{l}\text { Composition du } \\
\text { conseil } \\
\text { d'administration }\end{array}$ & $\begin{array}{l}\text { Un collège des membres } \\
\text { de droit (15 } \\
\text { personnalités politiques } \\
\text { régionales) et un collège } \\
\text { des adhérents divisé en } \\
7 \text { sous-collèges. }\end{array}$ & $\begin{array}{l}4 \text { collèges : } \\
\text { - PME (10 représentants), } \\
\text { - Groupe } \\
\text { représentants), } \\
\text { - Ecole (3 représentants), } \\
\text { - Laboratoire } \\
\text { représentants). }\end{array}$ & $\begin{array}{l}\text { Deux collèges : } \\
\begin{array}{lr}\text { - Collège A dédié aux } \\
\text { entreprises }\end{array} \\
\text { représentants), } \\
\quad \text { Collège } \\
\begin{array}{lr}\text { - Beprésentant } \\
\text { universités et }\end{array} \\
\text { laboratoires les } \\
\text { représentants). }\end{array}$ \\
\hline $\begin{array}{l}\text { Participation des } \\
\text { acteurs du jeu vidéo } \\
\text { à la gouvernance }\end{array}$ & $\begin{array}{l}\text { Association Game In } \\
\text { représentant les acteurs } \\
\text { du jeu vidéo au sein du } \\
\text { sous-collège Entreprises }\end{array}$ & 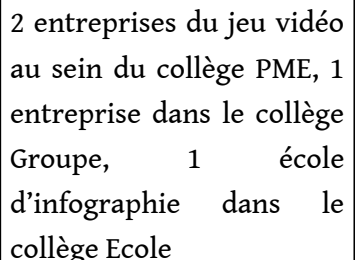 & $\begin{array}{l}1 \text { entreprise } \mathrm{du} \text { jeu } \\
\text { vidéo participant au } \\
\text { collège } \mathrm{A}\end{array}$ \\
\hline
\end{tabular}

Réalisation : B. Lusso, 2017, TVES, Lille 1, d'après les sites internet de Pictanovo (http:// www.pictanovo.com/), d'Imaginove (http://www.imaginove.fr/) et du PRIMI (http://www.primi.pro/fr)

Si la part des entreprises du jeu vidéo adhérant aux pôles transmédia est globalement limitée (9 à $10 \%$ à Lille et Marseille, 18 \% à Lyon), elles sont néanmoins portées par des motivations clairement économiques. Pour ces firmes, l'adhésion à l'association leur permet d'avoir une visibilité plus forte (lors des salons) et de trouver plus facilement de nouveaux clients. D'autres reconnaissent les bienfaits de ces structures d'accompagnement en termes d'innovation technologique et de performance économique (Lusso, 2014). Quelques structures de formation spécialisées dans le design numérique ou l'infographie adhèrent au pôle (Supinfogame, Pole IIID pour Pictanovo, Bellecour Ecole pour Imaginove, ARIES pour le PRIMI...). Pour ces écoles, la participation au pôle leur donne une place privilégiée pour observer l'évolution des métiers et identifier les besoins nouveaux des firmes, ce qui leur permet d'adapter l'offre de formation aux besoins du monde entrepreneurial. Les écoles cherchent à attirer des professionnels du jeu vidéo susceptibles d'intervenir dans leur formation, mais aussi à favoriser une meilleure insertion de leurs étudiants dans le monde du travail. Ces structures de formation encouragent à leur tour les laboratoires de recherche qui leur sont parfois associés à adhérer au pôle, pour favoriser un meilleur transfert de l'innovation vers le monde entrepreneurial (Lusso, 2014).

Les acteurs du jeu vidéo participent activement au conseil d'administration et donc, à la gouvernance stratégique du pôle. Si une seule entreprise marseillaise est représentée au conseil d'administration du PRIMI, la présence des firmes productrices de contenus vidéoludiques est plus importante au sein du pôle lillois Pictanovo. Le président de l'association Game In dirige le sous-collège Entreprises du conseil d'administration de Pictanovo. A Lyon, les acteurs de l'industrie du jeu vidéo participent activement à la gouvernance du pôle transmédia : trois entreprises et une école participent au conseil d'administration d'Imaginove. Cette implication dans la vie du pôle de compétitivité témoigne d'un fort rapprochement des acteurs du jeu vidéo. Dès le début des années 2010 sont mis en place des réunions, des formations et des partenariats entre les acteurs de l'industrie du jeu vidéo de la région Rhône-Alpes. Ce rapprochement est 
formalisé par la création en 2015 d'une nouvelle association nommée Only Games qui regroupe d'après son site officiel 40 adhérents en 2017. L'objectif de ce club d'entreprises est de renforcer en dehors du cadre d'Imaginove la coopération entre les développeurs de jeux vidéo de la région.

Si les pouvoirs publics ne sont pas forcément directement représentés au sein du conseil d'administration des associations professionnelles transmédia, ils financent néanmoins leurs actions. Le rapport d'activité de Pictanovo pour l'année 2016 annonce une subvention de la région des Hauts-de-France (qui a pris la suite de la Région NordPas-de-Calais) d'un montant total de 7,5 millions d'euros pour l'année 2017 et divisée en sept fonds distincts. Parmi ces fonds, 600000 euros sont alloués à l'industrie du jeu vidéo. Quant au PRIMI, son rapport d'activité pour l'année 2016 n'oublie pas de mentionner ses principaux partenaires financiers qui sont en fait les institutions publiques étatique, régionale (région PACA), départementale (département des Bouches du Rhône) et métropolitaine (métropole Aix-Marseille Provence). Enfin, il ne faut pas oublier qu'Imaginove est un pôle de compétitivité et reçoit des financements publics plus importants. Sur les 11,4 millions d'aides au financement d'Imaginove présentées dans le rapport d'activité 2016 de l'association, 6 millions sont issus de l'État français (Fonds unique interministériel, Agence nationale de la recherche), le reste provenant essentiellement de la région Auvergne-Rhône-Alpes (qui a pris la suite de la suite de la région Rhône-Alpes) et de la métropole de Lyon. Les frais d'adhésion aux différents pôles ne représentent qu'une source de financement mineure. Ce sont bien les pouvoirs publics qui assurent l'essentiel du financement des associations professionnelles transmédia et de leurs actions collectives.

En une quinzaine d'années, l'industrie du jeu vidéo des aires métropolitaines de Lille, Lyon et Marseille s'est progressivement structurée autour de clubs d'entreprises thématiques puis de vastes associations professionnelles transmédia œuvrant à l'échelle régionale. Le rôle des pouvoirs publics, notamment celui de l'État, des régions et des métropoles, est déterminant dans la structuration de cette industrie. Ils encouragent la construction de pôles transmédia et financent une bonne partie de leur activité. Les acteurs du jeu vidéo sont désormais intégrés aux associations professionnelles transmédia qui mettent en place des actions d'animation en direction de l'industrie vidéoludique.

\section{La construction de projets collaboratifs dans le cadre des pôles transmédia}

31 Pour les trois pôles, une stratégie de développement a été mise en place pour favoriser le développement de l'industrie du multimédia, et plus spécifiquement celle du jeu vidéo. On peut distinguer trois axes stratégiques :

- l'emploi et la formation,

- le soutien à la recherche collaborative avec le lancement d'appels à projets collaboratifs,

- le développement commercial et international des entreprises.

Intégrés à la stratégie des pôles, les acteurs du jeu vidéo sont parties prenantes des actions d'animation proposées par les associations professionnelles transmédia. L'enjeu de cette section est d'évaluer, à partir de quelques exemples, l'efficience de ces actions 
sur le renforcement des proximités géographique, sociale et cognitive au sein de l'industrie du jeu vidéo des aires métropolitaines de Lille, Lyon et Marseille.

\section{La question de la formation et de l'insertion professionnelle}

Préalable à une dynamique collaborative, le rapprochement entre les écoles et le monde entrepreneurial devient un enjeu clé des actions d'intermédiation des trois pôles. Au regard de l'offre de formation proposée dans les trois territoires analysés, le tissu entrepreneurial reste très étroit, ce qui rend particulièrement difficile l'insertion dans la vie professionnelle des jeunes diplômés formés dans la métropole en question. En 2011, environ la moitié des étudiants formés aux métiers de l'infographie quittaient les aires métropolitaines de Lyon et de Marseille, ce chiffre atteignait même les $70 \%$ pour l'ensemble de l'aire métropolitaine de Lille (Lusso, 2011). Le problème tient autant à l'offre inadaptée aux besoins réels des entreprises qu'à la faiblesse des débouchés professionnels dans chacune des trois aires métropolitaines. Les jeunes sont formés à Lille, à Lyon ou à Marseille et s'en vont là où ils peuvent trouver un emploi, principalement en Ile-de-France et en Amérique du Nord. Les trois aires métropolitaines souffrent d'une pénurie de talents dans les métiers de l'infographie et du jeu vidéo. De plus, les recrutements conjuguent désormais les deux dimensions, technique et créative, ce qui les rend plus difficiles à réaliser. Au moment de la création des pôles à la fin des années 2000, 70 \% des studios de développement de jeux vidéo rencontraient des difficultés de recrutement, notamment dans les domaines de la programmation, de l'infographie et du game design (Lusso, 2011).

Afin de remédier à ces difficultés, les trois associations professionnelles transmédia ont mis en place une série d'actions visant à améliorer l'insertion des jeunes créateurs. Certains pôles transmédia organisent des événements visant à faire connaitre les créateurs de contenus vidéoludiques. C'est le cas du Challenge Jeunes Talents, un concours lancé en 2013 par Pictanovo et ouvert aux étudiants de la région des Hauts-deFrance ayant une expérience dans la création d'images numériques et de jeux vidéo. Les nominés bénéficient d'un soutien à la promotion de leur projet. Des rendez-vous sont fixés avec les professionnels de l'image afin que ces derniers accompagnent les jeunes créateurs dans la diffusion de leur produit. Le projet de jeu vidéo Electro'Field, développé par quatre étudiants de l'école d'infographie roubaisienne Pole IIID et nominé lors de l'édition 2015 du Challenge Jeunes Talents, a été sélectionné par la Transmédia Immersive University, une association chargée d'accompagner les porteurs du projet dans la commercialisation de leur produit.

Différents dispositifs de formation sont mis en place. C'est le cas pour Imaginove de Gamagora, un cursus de formation initiale au jeu vidéo proposé par l'Institut de la Communication de Lyon 2 qui comprend deux diplômes universitaires de premier cycle et un master professionnel. La formation est dispensée à la fois par des universitaires et des professionnels du jeu vidéo. Ces derniers sensibilisent les étudiants à l'importance du travail collaboratif selon une approche transmédia. Durant leur formation, les étudiants apprennent l'écriture et la production d'un jeu vidéo. Puis, ils créent leur propre maquette de jeu vidéo dans les conditions d'un studio. Ces maquettes sont présentées et testées par le grand public et les professionnels de l'industrie du jeu vidéo durant le Gamagora Game Show. Par ces différentes actions, un rapprochement s'effectue entre les jeunes diplômés et les professionnels du jeu vidéo. 
L'insertion professionnelle des étudiants s'en trouve nettement améliorée. Entre 2011 et 2016, 250 jeunes formés dans le cadre de Gamagora ont trouvé un emploi dans la Région Rhône-Alpes. Le décloisonnement des frontières entre les structures de formation, les firmes et la main-d'œuvre créative est en route, ce qui favorise la mise en œuvre de coopérations diverses et le développement de programmes de recherche collaborative.

\section{La mise en place de programmes de recherche collaborative}

Les associations professionnelles transmédia des trois aires métropolitaines accordent dans leur stratégie d'animation une grande importance aux programmes de recherche collaborative. Dans le domaine du jeu vidéo, les jeux sérieux (serious games) constituent à la fin des années 2000 une priorité pour les pôles lillois et lyonnais. Le jeu sérieux est une application informatique qui combine une intention sérieuse de type pédagogique, informative, communicationnelle ou marketing, avec des ressorts ludiques issus d'un ou de plusieurs métiers du jeu vidéo (Alvarez et Rampoux, 2007). A la suite d'un appel à projets serious gaming lancé en 2009 par le secrétariat d'État à l'industrie numérique, 8 et 9 projets ont été respectivement retenus dans les aires métropolitaines de Lille et de Lyon qui ont souhaité prolonger cette dynamique porteuse de développement pour l'industrie du jeu vidéo.

Pictanovo a lancé dès 2009 son premier appel à projet serious game. Ce dispositif doté d'un budget de 550000 euros a favorisé la labellisation de 12 projets. Les entreprises participant à ce projet sont principalement des jeunes structures qui ont souvent vu le jour en même temps que l'association professionnelle transmédia, à l'instar de 3Dduo, Idées3Com ou CCCP. Ces firmes s'associent parfois à des centres de recherche pour réaliser ces projets. L'appel à projets a permis l'implantation sur Lille d'une équipe de la société parisienne KTM Advance qui figure parmi les leaders nationaux du jeu sérieux (27 emplois) et la création à Valenciennes en 2009 d'une nouvelle entreprise Byook (16 emplois). L'importance des retombées territoriales de cette initiative explique le lancement l'année suivante par Pictanovo d'un deuxième appel à projets serious game qui a abouti à la concrétisation de 12 nouveaux projets et à la création d'une cinquantaine d'emplois (Lusso, 2011). Le fonds destiné aux jeux sérieux a été intégré dès 2013 au fonds d'Expériences interactives doté d'un budget de 1,2 million d'euros. D'après le rapport d'activité de Pictanovo (2013), ce fonds a financé pour l'année 2013 un total de 47 projets, dont 14 jeux sérieux. Sur les 140 projets financés pour le Fonds d'Expériences interactives entre 2013 et 2016, un tiers a été consacré aux projets collaboratifs dans le domaine des jeux vidéo et des jeux sérieux. Cette forte implication des entreprises du jeu vidéo dans les projets collaboratifs du pôle explique le lancement en 2017 par Pictanovo du premier fonds régional d'aide au prototype et à la production de jeux vidéo.

Le développement de ces projets collaboratifs favorise le renforcement d'une proximité cognitive qui se traduit par un meilleur transfert des connaissances entre les laboratoires de recherche et les entreprises. L'analyse des dynamiques réticulaires existantes au sein des différents pôles lillois, lyonnais et marseillais montre que les entreprises ne demeurent pas enfermées dans des relations d'hyperproximité, mais qu'elles s'ouvrent aussi sur des acteurs extérieurs, principalement régionaux. C'est le cas du jeu vidéo de course automobile publié en 2014 et intitulé The Crew qui est le 
résultat d'un partenariat entre le studio lyonnais Ivory Tower pour la production et l'établissement grenoblois de l'éditeur français de jeux vidéo Ubisoft pour la distribution. Le succès du jeu a facilité le rachat en 2015 d'Ivory Tower par Ubisoft en vue de l'écriture d'une suite (The Crew 2) pour 2018. En rejoignant Ubisoft, les équipes d'Ivory Factory bénéficieraient de l'infrastructure, des outils et de la technologie d'Ubisoft et apporteraient en échange leur expertise dans le domaine de la narration. Dans les trois aires métropolitaines, des relations formelles et informelles s'établissent entre les différents acteurs du jeu vidéo. La proximité sociale entre les firmes est même renforcée lorsque celles-ci sont se sont développées après la création des pôles transmédia. Plusieurs cas en attestent dans l'aire métropolitaine de Lille. Les fondateurs $\mathrm{du}$ studio de développement 3Dduo et du créateur d'applications interactives Idées3com sont d'anciens étudiants de l'Université de Lille 1 qui ont lancé leur entreprise respective dans le cadre de l'incubateur universitaire MITI. Ces derniers collaborent étroitement, notamment dans le cadre du programme de recherche Sphere (Lusso, 2014).

Si l'échelle régionale demeure une échelle de référence dans les collaborations entre les acteurs de l'industrie du jeu vidéo des aires métropolitaines de Lille, Lyon et Marseille, ces derniers n'hésitent plus à développer des coopérations nationales et internationales. Ainsi, le studio de développement lyonnais Artefacts Studio a cherché des compétences et des savoir-faire complémentaires en établissant des partenariats avec des entreprises nationales (Ubisoft) ou internationales (Electronic Arts). En effet, ces firmes maîtrisent des outils tels qu'Unreal Engine Maya et Motion Builder, logiciels auxquels Artefacts Studio n'avait initialement pas accès. Grâce à ses collaborations, Artefacts Studio a pu développer les jeux Fuel pour Asobo ou Assassin Creed II pour Ubisoft. De même, la compétence en jeu sérieux du studio lyonnais a été renforcée à la suite d'un partenariat avec le producteur audiovisuel lyonnais La Cuisine aux Images qui dispose de savoir-faire beaucoup plus complets dans l'écriture d'un scénario (Lusso, 2011 ; Lusso, 2014).

\section{Le développement des entreprises}

Les entreprises à forte croissance demeurent marginales. De nombreuses TPE ou PME désireuses de contrôler l'ensemble des tâches effectuées au sein de l'entreprise, n'intègrent pas la croissance parmi leurs objectifs (Janssen, 2011). Pourtant, la société lilloise Ankama a lancé dès le milieu des années 2000 plusieurs implantations à l'étranger. Créée en 2006, la filiale Ankama Studio est chargée de réaliser des jeux pour le compte d'autres sociétés, notamment américaines. Des filiales d'Ankama s'installent au Japon en 2009 et au Canada en 2013. Des entreprises sont rachetées par Ankama comme le montre l'exemple de la société japonaise de jeux mobiles Wizcorp en 2014. Malgré tout, l'exemple d'Ankama reste très minoritaire. En raison de leur taille, les entreprises du jeu vidéo hésitent à se lancer dans la croissance de leurs effectifs ou dans un développement à l'international (Lusso, 2011).

41 Afin de compenser ces difficultés, les organisations professionnelles transmédia mettent l'accent sur l'accompagnement des firmes dans leur stratégie de développement commercial et international. Chaque année, les pôles organisent des événements autour du jeu vidéo. L'objectif est d'assurer une meilleure visibilité 
nationale et internationale aux acteurs du jeu vidéo et de stimuler la production régionale. Trois grandes familles d'événements peuvent être distinguées :

- Les conférences professionnelles qui permettent aux acteurs locaux du jeu vidéo de découvrir, grâce à des intervenants extérieurs, les dernières innovations en matière d'usage, de technologie ou même d'organisation entrepreneuriale. La question des jeux sérieux a été largement évoquée dans ces réunions durant la décennie 2000.

- Les festivals composés de concours ouverts aux talents régionaux et, le cas échéant, extérieurs à l'image des e-virtuoses, rencontres européennes des jeux sérieux organisées par Pictanovo. Ces événements cherchent à promouvoir la jeune création régionale vidéoludique.

- Les conventions d'affaires qui sont des moments d'échange en face à face pour les entreprises qui souhaitent commercialiser un produit, acquérir de nouveaux marchés nationaux ou internationaux (Serious Game Expo à Lyon), construire de nouveaux partenariats (Les e-créateurs à Lille) ou recruter de jeunes diplômés (Talent Day à Lyon).

Dans ce paysage, seule la Game Connection organisée à Lyon depuis 2001 semblait attirer un grand nombre d'entreprises extérieures à la région et avoir une dimension mondiale, dans la mesure où 42 pays étaient représentés parmi les 220 exposants du salon. Or, cette convention d'affaires a déménagé en 2011 de Lyon vers Paris. Désormais organisée en partenariat avec le cluster francilien Capital Games. En dépit du succès réel de cette initiative, il reste difficile pour une ville comme Lyon de conserver un événement d'une telle portée dans un pays aussi centralisateur que la France (Lusso, 2013).

La participation à des salons organisés à l'étranger est également proposée aux entreprises du jeu vidéo par les trois associations professionnelles. La proximité géographique est souvent privilégiée (participation aux salons allemands du jeu vidéo tels que la Game Developper Conference de Cologne pour les firmes adhérentes aux trois pôles), même si les salons organisés en Amérique du Nord (salon E3 à Los Angeles) font l'objet d'un intérêt croissant de la part des pôles lillois et lyonnais. C'est durant la Game Developper Conference de San Francisco que l'entreprise lyonnaise Artefacts Studio s'est rapprochée de l'entreprise américaine Electronic Arts pour mettre en place une coopération qui se traduira par la suite par un programme de recherche collaborative (Lusso, 2013). Enfin, les missions à l'international proposées par les pôles transmédia constituent un outil important pour le développement des entreprises productrices de contenus vidéoludiques. Le rapport d'activité de Pictanovo (2012) met en évidence le succès de mission export organisée à San Francisco en mars 2012 et ouverte à 4 PME régionales du jeu vidéo. Cette mission a été un succès réel permettant aux quatre firmes régionales participantes de rencontrer 85 acteurs du jeu vidéo et de construire des réseaux à l'échelle internationale.

\section{Conclusion}

En moins de vingt ans, les aires métropolitaines de Lille, Lyon et Marseille ont été marquées par le développement et la structuration de leur industrie du jeu vidéo selon une logique transmédia. Ces dynamiques résultent de l'action volontariste menée par les pouvoirs publics régionaux et métropolitains qui ont encouragé et financé la construction de clubs d'entreprises dédiés à l'industrie du jeu vidéo puis d'associations professionnelles régionales plus généralistes: Pictanovo pour la Région des Hauts-de- 
France, Imaginove pour Auvergne-Rhône-Alpes et PRIMI pour PACA. Elles regroupent à la fois les firmes, institutions politiques, structures d'enseignement et de recherche œuvrant dans les domaines de l'audiovisuel, de l'animation, du jeu vidéo, de la création de sites Internet ou des télécommunications. Par leur présence au sein du conseil d'administration des associations professionnelles, les acteurs du jeu vidéo participent à la vie du pôle et contribuent à la construction d'une véritable proximité institutionnelle avec l'ensemble des professionnels du multimédia.

La proximité organisationnelle au sein de l'industrie du jeu vidéo se traduit par des actions d'animation comme l'adaptation de l'offre de formation aux besoins des entreprises locales, l'organisation ou la participation aux salons professionnels dédiés aux jeux vidéo ou le lancement d'appels à projets. Les acteurs du jeu vidéo participent aux actions d'intermédiation des associations professionnelles transmédia. Un climat de confiance s'instaure et témoigne d'une proximité sociale grandissante qui dépasse les frontières de l'industrie du jeu vidéo. Par le développement de projets collaboratifs, les acteurs du jeu vidéo renforcent la coopération entre les firmes, mais aussi avec les écoles et les laboratoires de recherche, ce qui entrâne une meilleure diffusion des innovations scientifiques en direction $d u$ monde entrepreneurial. Enfin, les collaborations entre les acteurs du jeu vidéo se multiplient aux échelles locale, régionale (et dans une moindre mesure nationale et internationale) illustrant une véritable proximité géographique.

Jean-Claude Daumas (2007) distingue différents stades de vie des clusters et des districts industriels : émergence, structuration, déclin et, le cas échéant, renaissance. La construction de réseaux au sein, mais aussi en dehors du pôle transmédia, témoigne d'une structuration réelle de l'industrie du jeu vidéo dans les aires métropolitaines de Lille, Lyon et Marseille. Pour ces trois territoires, l'industrie du jeu vidéo s'inscrit dans une dynamique de construction de clusters innovants avec des collaborations plus ou moins avancées. Les mesures mises en œuvre par les pôles transmédia Pictanovo et Imaginove ont davantage concerné l'industrie vidéoludique et ont favorisé un rapprochement entre les acteurs du jeu vidéo. Malgré le développement d'une stratégie transmédia, le PRIMI est davantage resté une association de soutien à la production audiovisuelle expliquant la structuration plus lente de l'industrie du jeu vidéo régionale. Ces constatations rappellent que le cluster innovant n'a pas une vocation marchande (Potter and Doug-Watts, 2011), ce qui laisse présager une intervention de la puissance publique sur le long terme, dans la construction de réseaux multiscalaires. Or, dans un contexte de réduction des dépenses publiques, l'implication financière des régions et des métropoles est remise en cause, ce qui pousse les acteurs de l'industrie du jeu vidéo à chercher de nouvelles sources de financement, d'origine privée.

\section{BIBLIOGRAPHIE}

ALBERTI F. (2004), « The crisis of the industrial district of Como: a longitudinal analysis of its evolution », 20th Egos Colloquium, juillet, Ljubljana. 
ALVAREZ J. \& RAMPOUX O. (2007), « Serious game: just a question of posture? » Artificial \& Ambient Intelligence, AISB'07, Avril, pp. 420-423.

AUGROS J. (2012), « La ruée vers l'or. Nouveaux écrans, nouvelles recettes ? », Mise au point, 4-2012 (https://map.revues.org/634).

AZEMARD G. (2013), 100 notions pour le crossmédia et le transmédia, Charenton-le-Pont, éditions de l'immatériel.

BERTHINIER-PONCET A. (2014), « Gouvernance et dynamiques d'innovation au sein d'un technopôle : une analyse par les pratiques institutionnelles d'innovation », Management International, 19-1, pp. 94-112.

BOSCHMA R. (2004), « Proximité et innovation », Géographie rurale, 280, pp. 8-24.

BRENNAN J. \& MARTIN E. (2012), “Spatial proximity is more than just a distance measure”, International journal of human-computer studies, 70-1, pp. 88-106.

CHAMPENOIS C. (2012), "How can a cluster policy enhance entrepreneurship? Evidence from the German 'Bioregio' case", Environment and planning C : government and policy, 30-5, pp. 796-815 https://hal.archives-ouvertes.fr/hal-00936094.

CHANTEPIE P. \& LE DIBERDER A. (2012), Révolution numérique et industries culturelles, Paris, La Découverte.

COOKE P. (2001), "Regional innovation systems, clusters, and the knowledge economy", Industrial and corporate change, 10-4, pp. 945-974.

CORIS M. (2008), « Proximités et délocalisations : le cas du logiciel », Revue d'économie régionale et urbaine "La Proximité : 15 ans déjà", 3, pp. 361-380.

CREVOISIER O. (2001), «L'approche par les milieux innovateurs : état des lieux et perspectives », Revue d'économie régionale et urbaine, 1, pp. 153-166.

DAUMAS J-C. (2007), « Dans la boîte noire des districts industriels », in Daumas J-C., Lamard P., Tissot L., Les territoires de l'industrie en Europe (1750-2000), Entreprises, Régulations, Trajectoires, Besançon, Presses Universitaires de Franche-Comté, pp. 9-34.

DELGADO M., PORTER M.E. \& STERN S. (2014), "Clusters, convergence, and economic performance”. Research policy, 43, pp. 1785-1799.

DEPRET M-H. \& HAMDOUCH A. (2009), « Clusters, réseaux d'innovation et dynamiques de proximité dans les secteurs high-tech », Revue d'économie industrielle, 4-2009 https:// rei.revues.org/4067.

DUBOULOZ S. \& BOCQUET R. (2013), « Innovation organisationnelle. S'ouvrir pour innover plus ? ", Revue française de Gestion, 236, pp. 129-147.

ERNST D. (2006), “Innovation offshoring - Asias's emerging role in global innovation networks", East West special reports, 10

ETZKOWITZ H. \& LEYDERSDORFF L. (1997), Universities and the global knowledge economy : a Triple Helix of university-industry-government relations, London, Pinter.

ETZKOWITZ, H. \& RANGA M. (2011), “Spaces : a Triple Helix governance strategy for regional innovation”, in Rickne A., Laestadius S. \& Etzkowitz H. (eds), Innovation governance in an open economy : shaping regional nodes in a globalized world, London, Routledge.

FELDMAN M.P. (2003), “The locational dynamics of the US biotech industry : knowledge externalities and the anchor hypothesis", Industry and innovation, 10-3, pp. 311-328. 
GILLY J-P., KECHIDI M. \& TALBOT D. (2014), “Resilience of organizations and territories : the role of pivot firms", European management journal, 32-4, pp. 596-602.

GRANOVETTER M. (1985), "Economic action and social structure : the problem of embeddedness", American journal of sociology, 91, pp. 481-510.

HUBER F. (2011), « On the role and interrelationship of spatial, social and cognitive proximity : personal knowledge relationships of R\&D workers in the Cambridge IT Cluster ", Regional studies, pp. 1169-1182 https://hal.archives-ouvertes.fr/hal-00712360/document.

JANSSEN F. (2011), La croissance de l'entreprise. Une obligation pour les PME ?, Bruxelles, De Boeck.

LEDUCQ D. \& LUSSO B. (2011), « Le cluster innovant : conceptualisation et application territoriale ", Cybergéo : european journal of geography, Espace, société, territoire, article 521 https://cybergeo.revues.org/23513.

LUSSO B. (2011), Les dynamiques territoriales du secteur de l'image en mouvement dans les aires métropolitaines de Lille, de Lyon et Marseille, Thèse de doctorat, Université Lille 1.

LUSSO B. (2013), « Pôles transmédia, actions d'intermédiation et construction de liens au sein de l'industrie du multimédia des aires métropolitaines de Lille, Lyon et Marseille », Interventions économiques, 48-2013

LUSSO B. (2014), «Les facteurs d'émergence et de pérennisation du secteur de l'image en mouvement dans les aires métropolitaines de Lille, de Lyon et de Marseille », Territoire en mouvement, 23-24 https://tem.revues.org/2589.

MARTINUS K. \& SIGLER T.J. (2017), « Global city clusters: theorizing spatial and non-spatial proximity in inter-urban firm networks », Regional studies, pp. 1-13.

MENDEZ A., BARDET M. \& COURAULT B. (2008), « Quels systèmes de gouvernance pour les pôles de compétitivité constitués de PME : entre logiques d'intégration et de différenciation », AIMS, Nice, mai.

OCDE. (1999), Boosting innovation : the clusters approach, Paris, OECD.

PORTER M.E. (1998), « Clusters and the new economics of competition », Harvard business review, November-December 1998, pp. 77-90.

POTTER A. \& DOUG-WATTS H. (2011), « Evolutionary agglomeration theory: increasing returns, diminishing returns, and the industry life cycle », Journal of economic geography, 11-3, pp. 417-455.

RESBEUT M. \& GUGLER P. (2016), « Impact of clusters on regional economic performance: a methodological investigation and application in the case of the precision goods sector in Switzerland », Competitiveness review, 26-2, pp. 188-209.

SAXENIAN A.L. (1994), Regional advantage. Culture and cooperation in Silicon Valley and Route 128, Cambridge, Harvard University Press.

SAXENIAN A.L. (2006), The New Argonauts, regional advantage in a global economy, Cambridge, Harvard University Press.

SCOTT A. J. \& LERICHE F. (2005), « Les ressorts géographiques de l'économie culturelle : du local au mondial ", L'Espace Géographique, 34-3, pp. 207-222.

SNJV-IDATE (2016), Baromètre annuel du jeu vidéo en France (édition 2016), Paris, Idate-Digiworld http://www.snjv.org/barometre-annuel-du-jeu-video-en-france/.

STORPER M. (1997), The regional world: territorial development in a global economy, New York, Guilford. 
ZENTELIN J-L. (2015), « Mobilisation des proximités et gouvernance du cluster de Paris-Saclay », Revue d'Économie Régionale et Urbaine, n5, pp. 883-898.

\section{RÉSUMÉS}

Dans les aires métropolitaines de Lille, Lyon et Marseille, l'industrie du jeu vidéo n'était quasiment pas structurée. Les entreprises ne se connaissaient pas et ne collaboraient pas entre elles. À la fin des années 2000, trois associations professionnelles transmédia regroupant entreprises, écoles, centres de recherche et institutions publiques ont été lancées : Imaginove à Lyon, Pictanovo à Lille et le Pôle Régional de l'Image, du Multimédia et de l'Internet (PRIMI) à Marseille. Ces structures ont développé des actions d'animation et de médiation incitant aux collaborations entre les acteurs du jeu vidéo: création de plates-formes emploi/formation, lancement d'appels à projets collaboratifs, accompagnement au développement international des entreprises. Les premiers résultats montrent que les entreprises productrices de contenus vidéoludiques coopèrent activement avec les écoles et les laboratoires de recherche, principalement à l'échelle locale et régionale.

In the Lille, Lyon and Marseille metropolitan areas, the video games industry isn't really structured. Collaborations between firms are nonexistent. To improve this problematic situation, three cross-media professional associations have been created in the end of the 2000s : Imaginove in Lyon, Pictanovo in Lille and the Regional Image, Multimedia and Internet Cluster (PRIMI) in Marseille. These associations that aim at launching a cluster dynamic have developed several actions for animation and mediation : employment/training websites, calls for collaborative projects, international development missions. The first results show networks and collaborations between video games firms, schools, research centres and public authorities, primarily at the local and regional level.

\section{INDEX}

Mots-clés : industrie du jeu vidéo, entreprises, politiques publiques, cluster innovant, réseaux Keywords : video games industry, firms, public policies, innovative cluster, networks

\section{AUTEUR}

\section{BRUNO LUSSO}

Laboratoire TVES, université de Lille 